\title{
Metadata of a soil loss map to assess sediment delivery ratios of European river catchments
}

Andreas Gericke
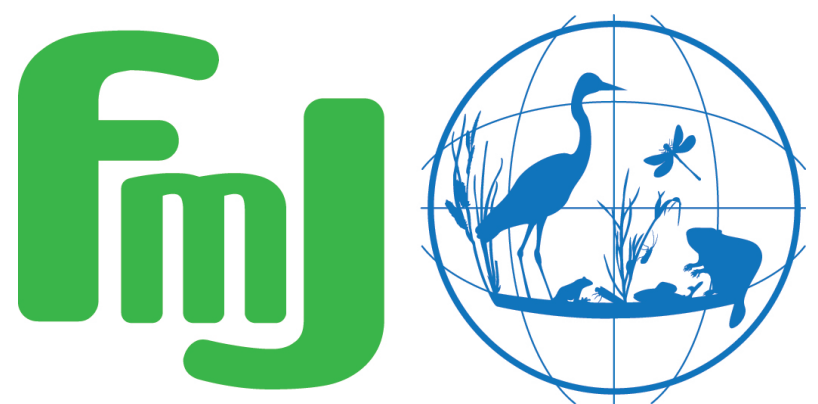

Freshwater Metadata Journal 



\section{Metadata of a soil loss map to assess sediment delivery ratios of European river catchments}

\section{Andreas Gericke ${ }^{1}$}

1 Leibniz-Institute of Freshwater Ecology and Inland Fisheries, Berlin, Germany; corresponding author: gericke@igb-berlin.de

Please cite this paper as follows: Gericke A., 2017. Metadata of a soil loss map to assess sediment delivery ratios of European river catchments. Freshwater Metadata Journal 20: 1-6.

https://doi.org/10.15504/fmj.2017.20

Received: 2017-02-27 / Published: 2017-03-06

\section{Keywords}

soil loss, universal soil loss equation

\section{Short description of the dataset/summary}

Various pan-European datasets and published empirical relationships were used to approximate 5 factors of the universal soil loss equation (USLE, Table 1 in the related publication). Different approaches for C and R were applied to consider different environmental conditions in northern and southern Europe (Gericke 2015, Fig. 1).

This soil-loss map (see Appendix) is the product of these factors: $\mathrm{E}=$ R.C.K.L.S with

L - slope-length factor, derived from the global SRTM digital elevation model (Jarvis et al. 2008)

$S$ - slope angle, calculated from SRTM

$\mathrm{R}$ - rainfall and runoff factor, taken from long-term average annual precipitation for European catchments (Vogt et al. 2007)

C - vegetation cover, derived from European and global data on land use and land cover (Corine Land Cover 2006, GlobCover v2.2) (European Environment Agency 2010, 2010a, 2010b, European Space Agency 2008) $\mathrm{K}$ - soil erodibility, derived from the European Soil Database (European Commission \& European Soil Bureau Network 2004)

The unit of $\mathrm{E}$ is tons/hectare. The spatial resolution of the gridded dataset (100 metres) was determined by the digital elevation model and the land use data.

In the related publication (Gericke 2015), this map was named "2kbc". It was compared to other realisations and found to be most suitable to explain the spatial variability of suspended-solids yields and sediment delivery ratios (SDR) of river catchments in various European regions.

This map can be used in regional to continental studies to obtain regional sediment delivery ratios or to estimate sediment 
yields of large river catchments. Users of this map should keep in mind the inherent limitations of the USLE and sediment yields, as well as the accuracy of the underlying datasets when assessing results. Please note that none of the alternative maps in the related study was found to optimally predict SDR and sediment yields everywhere.

\section{General information}

dataset entry ID:

name of the dataset:

full name of the dataset:

dataset short name:

type of dataset:

data type:
FWM_13

Soil loss map to assess sediment delivery ratios of European river catchments European soil loss map environmental characteristics database raster data (e.g. GeoTIFF)

science keywords according to GCMD:

topic:

Land Surface

keywords:

erosion, suspended solids, degradation

ISO topic category according to ISO 19115:

Environment, Geoscientific Information

\section{Technical and administrative specifications}

$\begin{array}{ll}\text { data format: } & \text { oth } \\ \text { others/details: } & \text { Erd } \\ \text { operating system: } & \text { all } \\ \text { current access level: } & \text { inte } \\ \text { currently available through GBIF: } & \text { no } \\ \text { exchange planned: } & \text { no } \\ \text { data in data repository: } & \text { no }\end{array}$

Do you plan to publish the data on the Freshwater Biodiversity Data Portal:

update level:

documentation:

type:

language:

others/details:

contact details:

metadata contact person:

first, last name:

phone:

email:

institution:

province, state:

country

web address:

technical contact person:

first, last name:

phone:

email: no

completed

scientific paper

English

see "dataset related references" below

Andreas Gericke

+493063924072

gericke@igb-berlin.de

Leibniz Institute of Freshwater Ecology and Inland Fisheries

Berlin

Germany

http://www.igb-berlin.de

Andreas Gericke

+493063924072

gericke@igb-berlin.de 
scientific contact person:

first, last name:

phone:

email:
Andreas Gericke

$+493063924072$

gericke@igb-berlin.de

\section{Intellectual property rights and citation}

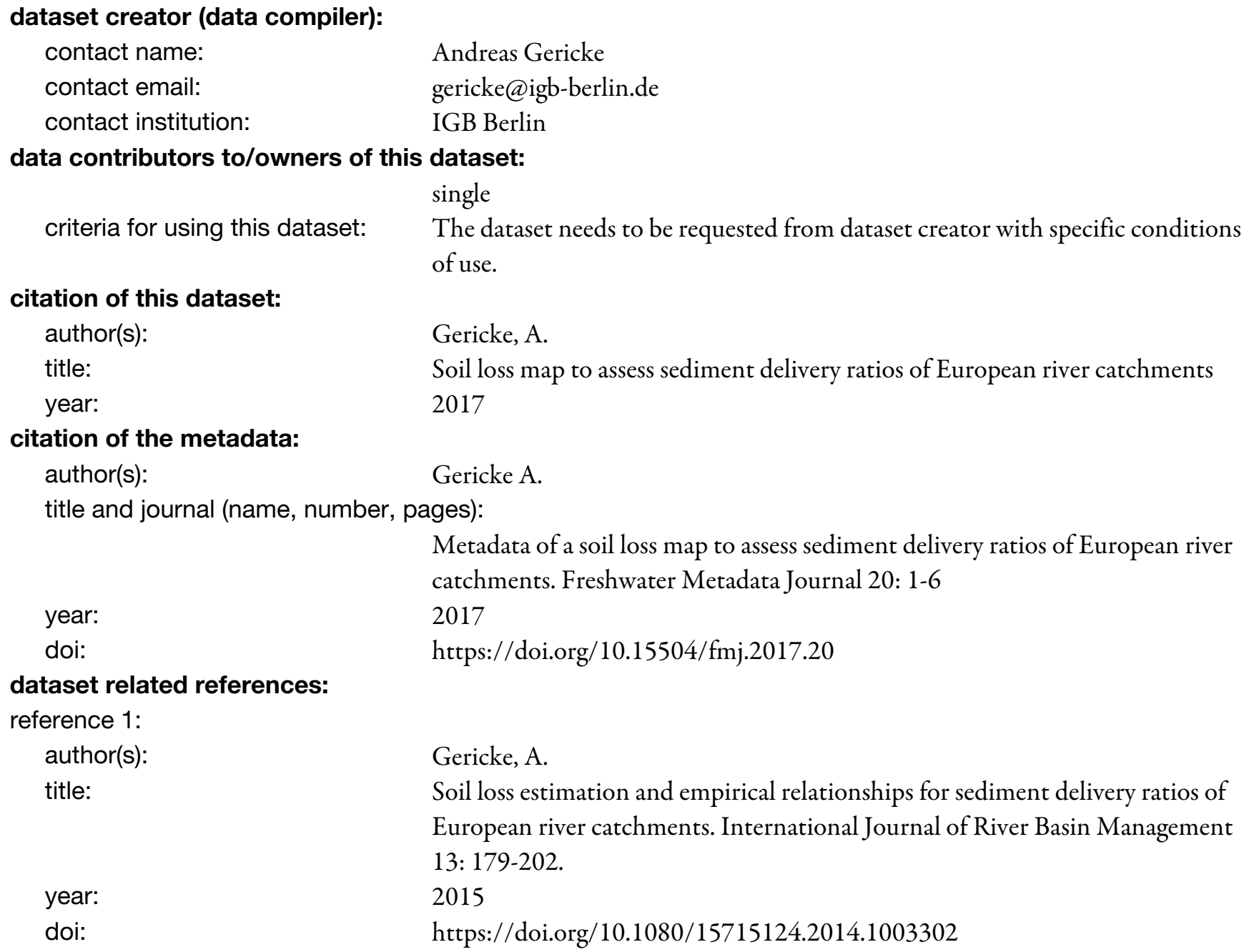

Metadata of a soil loss map to assess sediment delivery ratios of European river catchments. Freshwater Metadata Journal 20: 1-6

year: 2017

doi: $\quad$ https://doi.org/10.15504/fmj.2017.20

dataset related references:

reference 1:

author(s):

title:

Gericke, A.

Soil loss estimation and empirical relationships for sediment delivery ratios of European river catchments. International Journal of River Basin Management 13: 179-202.

year: 2015

doi: $\quad$ https://doi.org/10.1080/15715124.2014.1003302

\section{General data specifications}

regional coverage of the dataset: scale of the dataset:

continental

continents:

Europe

spatial extent (bounding coordinates):

southernmost latitude $\left[^{\circ}\right]$ : $\quad 35$

northernmost latitude $\left[{ }^{\circ}\right]: \quad 60$

westernmost longitude $\left[{ }^{\circ}\right]$ : $\quad-10$

easternmost longitude $\left[^{\circ}\right]:$

minimum altitude: $\quad 0$ metres

maximum altitude: $\quad 4000$ metres

countries: $\quad$ Europe: Albania, Austria, Belgium, Bosnia and Herzegovina, Bulgaria, Croatia,

Czech Republic, Denmark, Estonia, France, Germany, Gibraltar, Greece, 
ecosystem type:

comments:

\section{Site specifications}

coordinate system/grid data:

datum (e.g. WGS84):

grid data available:

resolution:

unit:

comments:
Hungary, Ireland, Italy, Latvia, Liechtenstein, Lithuania, Luxembourg, Macedonia, Moldova, Montenegro, Netherlands, Poland, Portugal, Romania, Serbia, Slovakia, Slovenia, Spain, Switzerland, Ukraine, United Kingdom, Kosovo, Pridnestrovie (Transnistria) general freshwater The modelled *average* soil loss was derived from the average precipitation 1975-1999 (CCM2) and land use maps for 2006 (CLC, GlobCover).

projected, others others: EPSG:3035

ETRS89

yes

100

$\mathrm{m}$

Definition of the coordinate system as well-known text:

PROJCS["ETRS89 / ETRS-LAEA",

GEOGCS["ETRS89",

DATUM["European_Terrestrial_Reference_System_1989", SPHEROID["GRS 1980",6378137,298.257222101, AUTHORITY["EPSG","7019"]],

AUTHORITY["EPSG","6258"]],

PRIMEM["Greenwich",0,

AUTHORITY["EPSG","8901"]],

UNIT["degree",0.01745329251994328, AUTHORITY["EPSG","9122"]],

AUTHORITY["EPSG","4258"]],

UNIT["metre",1,

AUTHORITY["EPSG","9001"]],

PROJECTION["Lambert_Azimuthal_Equal_Area"],

PARAMETER["latitude_of_center",52],

PARAMETER["longitude_of_center",10],

PARAMETER["false_easting",4321000],

PARAMETER["false_northing",3210000],

AUTHORITY["EPSG","3035"],

AXIS["X",EAST],

AXIS["Y",NORTH]]

\section{Climate and environmental data}

climate related data:

physico-chemistry data: no data available

no parameter data per catchment available

no parameter data per site avaiable

no data available 


\section{Other specifications}

\section{GIS layers, shapes related to the dataset:}

land use

environmental variables (freshwater or terrestrial)

climatic variables (current and predictions)

others/specify

others (specify):

availability of photos:

availability of maps:

digital elevation model

no

no

quality control procedures:

Were any quality control procedures applied to your dataset?

comments: no

The average soil loss is in tons per hectare per grid cell. It was derived from elevation (Jarvis et al. 2008), land use and land cover in 2006 (European Environment Agency 2010, 2010a, 2010b, European Space Agency 2008), soil data (European Commission \& European Soil Bureau Network 2004), and average annual precipitation 1975-1999 (Vogt et al. 2007).

European Commission \& European Soil Bureau Network 2004. The European Soil Database distribution. Version 2.0 [CD-ROM] EUR 19945 EN. http://esdac.jrc.ec.europa.eu/content/european-soil-database-v20-vector-and-at tribute-data

European Environment Agency 2010. Corine Land Cover 1990 raster data. Version 13.

http://www.eea.europa.eu/data-and-maps/data/ds_resolveuid/8b12c16a3c93ee c2d6944abe $42388 \mathrm{~d} 90$

European Environment Agency 2010a. Corine Land Cover 2000 raster data. Version 13.

http://www.eea.europa.eu/data-and-maps/data/ds_resolveuid/b00116e51c798 $65 \mathrm{cf} 89 \mathrm{a} 84162 \mathrm{~b} 8 \mathrm{fd} 21 \mathrm{e}$

European Environment Agency 2010b. Corine Land Cover 2006 raster data. Version 13.

http://www.eea.europa.eu/data-and-maps/data/ds_resolveuid/a645109f7a11d4 $3 \mathrm{f} 5 \mathrm{~d} 7 \mathrm{e} 275 \mathrm{~d} 81 \mathrm{f} 35 \mathrm{c} 61$

European Space Agency 2008. GLOBCOVER, ESA Globcover Project, led by MEDIAS-France/POSTEL. Version 2.2.

http://due.esrin.esa.int/page_globcover.php

Jarvis A., Reuter H. I., Nelson A. \& Guevara E. 2008. Hole-filled SRTM for the globe. Version 4. Available from: CGIAR-CSI SRTM 90 m Database.

http://srtm.csi.cgiar.org 
Vogt J., Soille P., de Jager A., Rimaviciute E., Mehl W., Foisneau S., Bodis K., Dusart J., Paracchini M-L., Haastrup P., Bamps C. 2007. A pan-European river and catchment database. Luxembourg: Office for Official Publications of the European Communities.

http://ccm.jrc.ec.europa.eu/php/index.php?action=view\&id=23

\section{Acknowledgements}

The help of C. Bosco (Joint Research Center, Ispra) with the European Soil Database is much appreciated.

\section{References}

European Commission \& European Soil Bureau Network, 2004. The European Soil Database distribution. Version 2.0 [CD-ROM] EUR 19945 EN.

http://esdac.jrc.ec.europa.eu/content/european-soil-database-v20-vector-and-attribute-data

European Environment Agency, 2010. Corine Land Cover 1990 raster data. Version 13.

http://www.eea.europa.eu/data-and-maps/data/ds_resolveuid/8b12c16a3c93eec2d6944abe42388d90

European Environment Agency, 2010. Corine Land Cover 2000 raster data. Version 13.

http://www.eea.europa.eu/data-and-maps/data/ds_resolveuid/b00116e51c7 9865cf89a84162b8fd21e

European Environment Agency, 2010. Corine Land Cover 2006 raster data. Version 13.

http://www.eea.europa.eu/data-and-maps/data/ds_resolveuid/a645109f7a1 1d43f5d7e275d81f35c61

European Space Agency, 2008. GLOBCOVER, ESA Globcover Project, led by MEDIAS-France/POSTEL. Version 2.2. http://due.esrin.esa.int/page_globcover.php

Gericke A., 2015. Soil loss estimation and empirical relationships for sediment delivery ratios of European river catchments. International Journal of River Basin Management 13: 179-202.

https://doi.org/10.1080/15715124.2014.1003302

Jarvis A., Reuter H. I., Nelson A. \& Guevara E., 2008. Hole-filled SRTM for the globe. Version 4. Available from: CGIAR-CSI SRTM 90 m Database, http://srtm.csi.cgiar.org

Vogt J., Soille P., de Jager A., Rimaviciute E., Mehl W., Foisneau S., Bodis K., Dusart J., Paracchini M-L., Haastrup P., Bamps C., 2007. A pan-European river and catchment database. Luxembourg: Office for Official Publications of the European Communities. http://ccm.jrc.ec.europa.eu/php/index.php?action=view\&id=23 
Appendix 
The appendix shows a map of the dataset (Fig. 1).

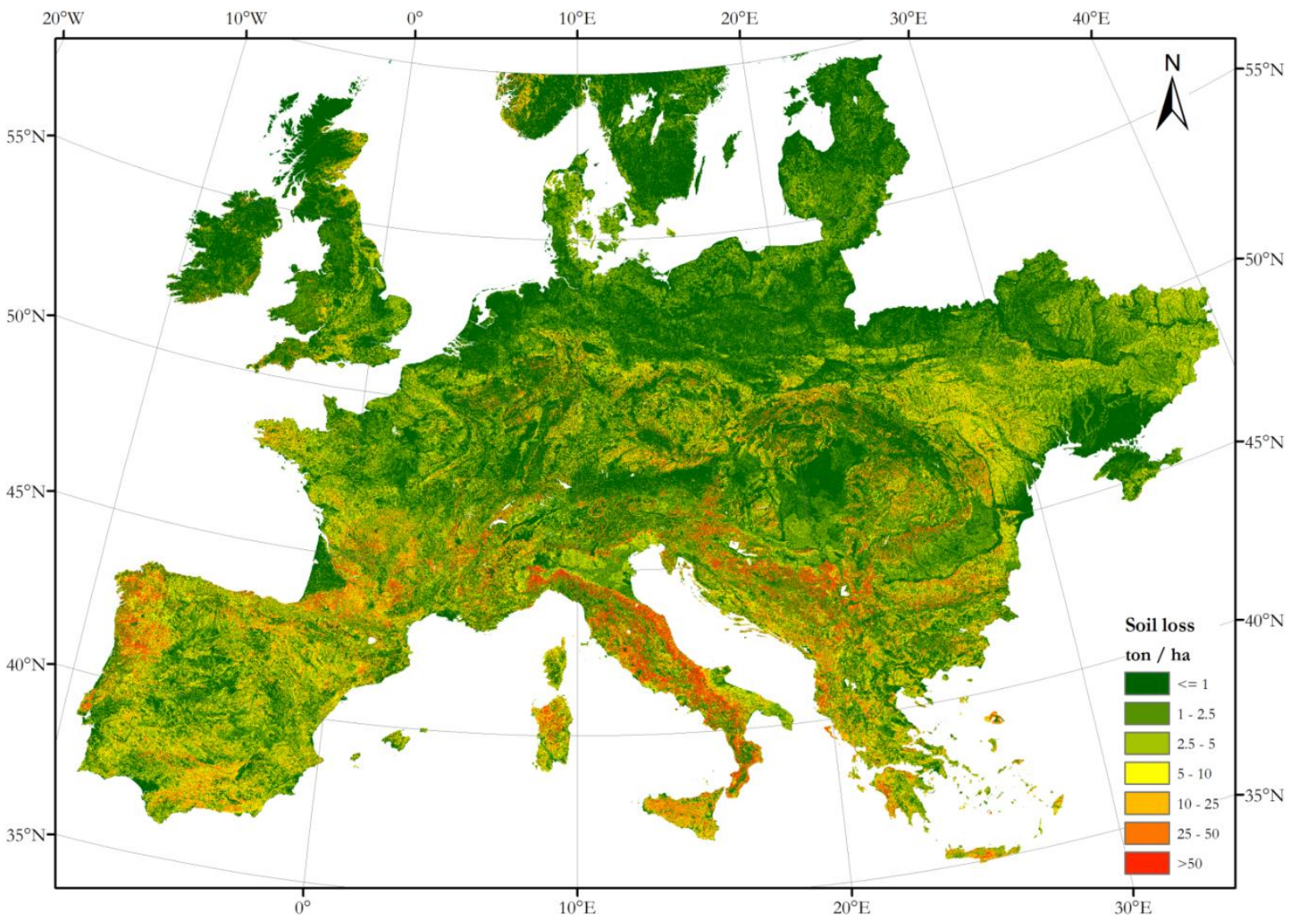

Fig. 1. Soil loss in tons per hectare. Missing values in white for some non-erosional areas (lakes, seas) and some countries (Andorra, Belarus, Iceland, Russia, San Marino, Turkey, and Vatican City). 